\title{
Controlling Gas-Phase Reactions for Efficient Charge Reduction Electrospray Mass Spectrometry of Intact Proteins
}

\author{
Brian L. Frey, Yuan Lin, Michael S. Westphall, and Lloyd M. Smith \\ Department of Chemistry, University of Wisconsin-Madison, Madison, Wisconsin, USA
}

\begin{abstract}
Charge reduction electrospray mass spectrometry (CREMS) reduces the charge states of electrospray-generated ions, which concentrates the ions from a protein into fewer peaks spread over a larger $\mathrm{m} / \mathrm{z}$ range, thereby increasing peak separation and decreasing spectral congestion. An optimized design for a CREMS source is described that provides an order-ofmagnitude increase in sensitivity compared to previous designs and provides control over the extent of charge reduction. Either a corona discharge or an $\alpha$-particle source was employed to generate anions that abstract protons from electrosprayed protein cations. These desired ion/ion proton transfer reactions predominated, but some oxidation and ion-attachment reactions also occurred, leading to new peaks or mass-shifted broader peaks while decreasing signal intensity. The species producing these deleterious side-reactions were identified, and conditions were found that prevented their formation. Spectrometer $\mathrm{m} / \mathrm{z}$ biases were examined because of their effect upon the signal intensity of higher $\mathrm{m} / \mathrm{z}$ charge-reduced protein ions. The utility of this atmospheric pressure CREMS was demonstrated using a cell lysate fraction from E. coli. The spectral simplification afforded by CREMS reveals more proteins than are observed without charge reduction. (J Am Soc Mass Spectrom 2005, 16, 1876-1887) (C) 2005 American Society for Mass Spectrometry
\end{abstract}

$\mathrm{M}$ ass spectrometric analysis of intact proteins has been practiced routinely since the advent of two soft ionization techniques for large molecules: matrix assisted laser desorption ionization (MALDI) [1, 2] and electrospray ionization (ESI) [3, 4]. Each of these techniques has advantages for different situations and often they are complementary in terms of which proteins ionize well. A primary difference between the two techniques is that MALDI generates ions in one or two low charge states, whereas ESI tends to create protein ions in a number of different and relatively high charge states. In fact, electrospray tends to put most proteins into the same $\mathrm{m} / \mathrm{z}$ range of the spectrum [5-7] (ca. 500-2500). While advantageous in some circumstances, this phenomenon can limit the ability to analyze protein mixtures by ESI due to spectral congestion. Such mixtures might comprise several truly distinct proteins or may involve heterogeneity of a "single" protein attributable to combinations of splice variants and post-translational modifications. In the latter case, this microheterogeneity means that the sample contains many similar species with slightly different molecular weights, which lead to broader peaks for each charge state and consequently increase

Published online September 28, 2005

Address reprint requests to Dr. L. M. Smith, Department of Chemistry, University of Wisconsin-Madison, 1101 University Avenue, Madison, WI 53706, USA. E-mail: smith@chem.wisc.edu probability of peak overlap. One way to avoid overlapping peaks from mixtures is with very high resolving power, and Fourier transform spectrometers have provided very good results in this regard [8-10]. Another method is to decrease the number of charge states for each analyte and to spread out these charge state peaks on the $\mathrm{m} / \mathrm{z}$ scale, both of which can be accomplished by reducing the charge, $z$, of the protein ions. Ideally, this charge reduction technique would be amenable to any type of mass analyzer, subject only to mass range limitations, and would maintain or enhance ESI's gentle ionization as well as its suitability for online analysis after separation.

Charge reduction of electrosprayed ions has been performed using a few different strategies [11-13]. ESI of proteins typically employs positive-ion mode whereby the proteins acquire charge by protonation of their basic residues. Thus, the most straightforward route to charge reduction would be a proton transfer reaction (PTR). Several researchers used strong neutral bases and succeeded in removing a few protons from the protein ions [14-16]. In contrast to these ion/ molecule reactions, McLuckey and coworkers have utilized ion/ion reactions to achieve extensive and controllable charge reduction $[11,13,17]$. Their method involves modification of a quadrupole ion trap instrument to inject and hold gas-phase anions simultaneously in the trap with the protein cations. They have exploited this strategy for a number of elegant studies 
and applications [11, 13, 18]. A few years ago, our group reported using ion/ion reactions for atmospheric pressure charge reduction electrospray mass spectrometry (CREMS) of biopolymers [19-22]. Either a radioactive $\alpha$-particle source $[19,20]$ or a corona discharge [21, 22] was employed to generate ions for the gas-phase proton transfer reactions.

In this paper, we explore the issues of protein oxidation, adduct formation, and possible ion losses occurring during CREMS to reach an understanding that has led to efficient control over the charge reduction technique. An optimized design is presented for a small charge reduction device that is easily fabricated and attached to the inlet of nearly any ESI-compatible mass spectrometer. With slight modification, the device allows for either a corona discharge or $\alpha$-particle source to generate the gas-phase anions, and we compare and contrast these two types of sources. The extent of charge reduction is easily tuned, which helps to separate overlapping peaks while working within the $\mathrm{m} / \mathrm{z}$ constraints of the mass analyzer. Furthermore, we have developed an understanding of oxidation and adduction reactions that can occur during charge reduction. This understanding allowed us to find conditions that avoid these types of deleterious side-reactions. The CREMS technique is shown here to be suitable for analysis of mixtures of large intact proteins, as is demonstrated for an unknown protein mixture derived from a cell lysate.

\section{Experimental}

\section{Chemicals and Samples}

Insulin (bovine) and bradykinin were obtained from Sigma-Aldrich (St. Louis, MO) and used without further purification. Samples were dissolved in a buffer of 1:1 $\mathrm{H}_{2} \mathrm{O}: \mathrm{MeOH}$ containing $1 \%$ acetic acid to a concentration of 3 or $10 \mu \mathrm{M}$. Platinum wire $(99.95 \%, 0.368 \mathrm{~mm}$ diameter) was purchased from Alfa Aesar (Ward Hill, MA). Burdick and Jackson (Muskegon, MI) HPLCgrade water, methanol, and acetonitrile were used as solvents and as sources of the corresponding vapors. Compressed nitrogen and USP-grade medical air were obtained from Linde Gas (Wilmington, DE). A protein sample fraction from an ion-exchange and reversephase separation of an E. coli cell lysate was used as received from collaborators, Dave Robinette and Morgan Giddings, at the University of North CarolinaChapel Hill. This sample was electrosprayed from a 1:1 $\mathrm{H}_{2} \mathrm{O}$ :ACN buffer containing $0.1 \%$ formic acid.

\section{CREMS Source Construction}

The CREMS sources shown in Figure 1 are small devices $(\sim 4.5 \mathrm{~cm}$ long) that mount directly to the mass spectrometer inlet, and therefore are referred to as nozzle extensions. Prior work with charge reduction employed a rather large chamber sitting in front of the
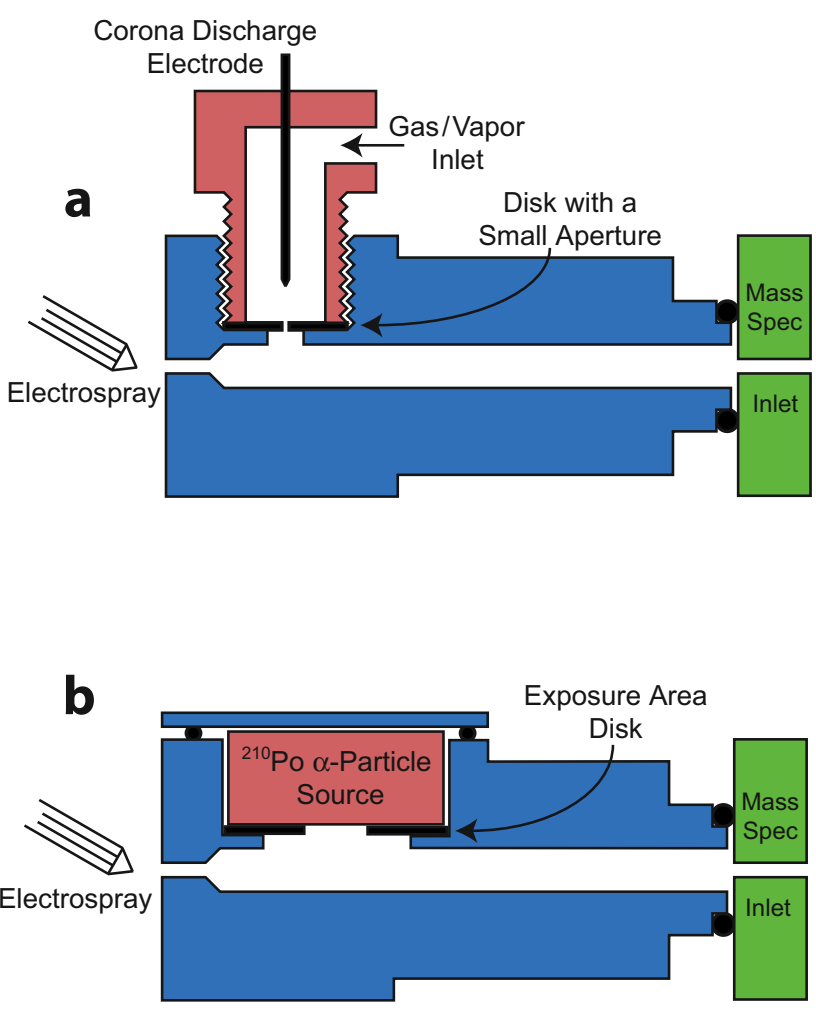

Figure 1. Diagrams of the corona discharge (a) and $\alpha$-particle (b) nozzle extension devices employed for charge reduction electrospray mass spectrometry (CREMS). Attachment to the mass spectrometer inlet via an o-ring seal greatly improves ion transmission. Control over the extent of charge reduction is provided by either (1) the gas flow rate through the corona discharge source or (2) the exposure area of the $\alpha$-particle source.

nozzle, and it required high flow rates of bath gas to transport ions through the chamber [20,21]. In contrast, the new design has an o-ring seal that leads to a vacuum-induced air-flow, which obviates the need for bath gas. For the corona discharge device (Figure 1a), another improvement was removing the corona discharge from the path of the electrospray ions. These changes in design improved transmission of the electrospray ions by nearly an order of magnitude.

The charge reduction chambers were machined from stainless steel (dark gray/blue components in Figure 1). The entrance hole for electrospray ions was $0.9 \mathrm{~mm}$ diameter for the first $3 \mathrm{~mm}$, and $2.5 \mathrm{~mm}$ diameter for the remaining $42 \mathrm{~mm}$ of the chamber. These dimensions should cause minimal pressure drop through the chamber because the majority of the pressure decrease will occur at the smaller nozzle orifice of the mass spectrometer $(0.35 \mathrm{~mm}$ diameter $\times 20 \mathrm{~mm}$ long) [23]. This orifice defines the gas flow rate $(G)$ through the charge reduction chamber according to the equation: $G=0.445 \mathrm{naD}^{2}$ where $n$ and $a$ are the number density and speed of sound for nitrogen gas $\left(2.5 \times 10^{19}\right.$ molecules $/ \mathrm{cm}^{3}$ and $3.5 \times 10^{4} \mathrm{~cm} / \mathrm{s}$, respectively), and $D$ is the orifice diameter $[23,24]$. The resulting $4.8 \times 10^{20}$ molecules/s, or $19 \mathrm{~cm}^{3} / \mathrm{s}$, yields a linear flow velocity of $390 \mathrm{~cm} / \mathrm{s}$, which corresponds to a residence time of $10 \mathrm{~ms}$ in the 
charge reduction chamber. The important result from these calculations is this $10 \mathrm{~ms}$ reaction time between electrospray ions and corona discharge generated ions.

Although functionally accurate, the light gray/red component in the top diagram is simplified somewhat from the actual design, which was assembled from mostly off-the-shelf components: a 1/4-28 polyetheretherketone (PEEK) Tee, a short piece of PEEK tubing, a fluorinated ethylene-propylene (FEP) sleeve for holding the $\mathrm{Pt}$ wire, as well as a few ferrules and PEEK fittings, all from Upchurch (Oak Harbor, WA). A PEEK bulkhead union (3/8-24 external and 1/4-28 internal threads) was modified slightly by removing the internal threads in the lower section so that the corona discharge plasma would be farther from the plastic. The tip of the $\mathrm{Pt}$ wire residing in this lower section was positioned 3 $\mathrm{mm}$ above a stainless steel disk ( $0.5 \mathrm{~mm}$ thick) with a 0.4 $\mathrm{mm}$ diameter hole in the center. The platinum wire was sharpened to a point $(\sim 10 \mu \mathrm{m})$ using a rotary grinder, followed by polishing with $3 \mu \mathrm{m}$ alumina powder. This corona discharge electrode was held at a large negative potential with respect to the stainless steel aperture disk, using a high voltage power supply (Stanford Research Systems, Sunnyvale, CA) and a $22 \mathrm{M} \Omega$ current-limiting resistor in series. The corona discharge voltage and current depended upon the gaseous medium; typical values were $-3200 \mathrm{~V}$ and $25 \mu \mathrm{A}$ in air or $-2400 \mathrm{~V}$ and $60 \mu \mathrm{A}$ in $\mathrm{N}_{2}$. After prolonged use, the aperture disks acquired a brown-black residue, as observed by others [25]; consequently, they were designed for easy removal to polish or replace them. Having an aperture in this disk is a small modification to the conventional point-to-plane configuration of a corona discharge [26], and it allows gas flow through the corona discharge, as discussed below.

The second charge reduction chamber (Figure 1b) employed a $5 \mathrm{mCi}^{210} \mathrm{Po} \alpha$-particle source (Model P-2042, NRD, Grand Island, NY). In this case, a large area of $\alpha$-particle exposure was sometimes necessary, rather than a small aperture. Machining a larger hole in the stainless steel created a short $(2.5 \mathrm{~mm}$ deep $)$ and wide (18 $\mathrm{mm}$ diameter) cylindrical chamber for the interaction of anions with electrosprayed cations. Ion losses in this chamber may account for a somewhat lower signal intensity compared to the corona discharge CREMS source. Controlling the amount of charge reduction was accomplished by placing a $0.5 \mathrm{~mm}$-thick stainless steel disk in front of the $\alpha$-particle source; holes in the disks varied in size from 0.8 up to $14.5 \mathrm{~mm}$ yielding exposure areas from 0.2 up to $64 \%$.

\section{CREMS Source Operation}

Since ESI of proteins is commonly performed in positive-ion mode, a "negative" corona discharge was utilized for anion generation [26, 27]. In general, a corona discharge ionizes the gaseous medium, thereby creating a plasma that contains positive ions and free electrons. By holding the sharp tip at a negative potential, most positive ions are annihilated after following the intense electric field lines toward this cathode. The electrons travel in the opposite direction and some of them become "thermalized" from numerous collisions with molecules. Subsequently, these electrons may attach themselves to molecules, and in air the most frequent result is the $\mathrm{O}_{2}^{-}$ion because it is more stable than $\mathrm{N}_{2}^{-}$. After chemical reactions in the plasma, other anionic species are sometimes produced as well (e.g., $\mathrm{O}_{3}^{-}, \mathrm{NO}_{2}^{-}$, $\mathrm{NO}_{3}^{-}, \mathrm{CO}_{3}^{-}$) depending on the gaseous medium [28-30]. In fact, this sequence of events is what leads to ionization of solvent or analyte molecules during atmosphericpressure chemical-ionization (APCI) mass spectrometry, which routinely employs a corona discharge [27, 29].

The anions created by the corona discharge were transported through the disk aperture into the path of the electrosprayed ions, which were en route to the mass spectrometer inlet. A simple gas flow meter (Dwyer Instruments, Michigan City, IN) was used to change the flow rate of gas through this aperture, and thereby provide control over the amount of charge reduction. Adding a vapor to the flowing gas was accomplished by bubbling the air or nitrogen through the appropriate solvent. [Potential Hazard: while a corona discharge is unable to ignite the organic vapor in air, there is a small risk of electrical arcing. We were careful to obtain a stable corona before flowing in the organic vapor. Employing nitrogen gas instead of air ensures further safety, and it actually produces more favorable results, as shown in the Results section.]

\section{Mass Spectrometry and Data Analysis}

All experiments were conducted with a Mariner orthogonal acceleration time-of-flight (oa-TOF) mass spectrometer (Applied Biosystems, Foster City, CA). This ESI-TOF instrument provides reasonable mass accuracy, resolution, and a wide $m / z$ range $(50-25,000)$ for charge reduced protein spectra. We have also attached the charge reduction device to an ion trap instrument (LCQ XP+, ThermoFinnigan, San Jose, CA) and that work will be published elsewhere. All samples, regardless of whether charge reduction was performed, were electrosprayed from a fused silica capillary $(90 / 20 \mu \mathrm{m}$ o.d./i.d., Polymicro Technologies, Phoenix, AZ). The samples were infused at a flow rate of $\sim 50 \mathrm{~nL} / \mathrm{min}$ by employing positive air pressure [20], although a syringe pump would be suitable as well. The spray tip potential of $\sim 2700 \mathrm{~V}$ was applied via a platinum wire immersed in the sample solution. Spectra of known proteins were obtained as ten co-added scans of $10 \mathrm{~s}$ each (unless otherwise noted). The cell lysate spectra were acquired as fifty co-added scans of 10 or 20 s each, without and with charge reduction, respectively.

Mass spectra were smoothed with Data Explorer software (Applied Biosystems); the number of data points used in the Gaussian smoothing algorithm was chosen to improve signal-to-noise without artificially broadening the peaks (3-99 points; always an equal 
number for spectra being directly compared). The Mariner instrument employs an ion counting detection method, and so the best quantitative measure is the sum of the ion counts for each data point across a peak. This ion count sum, $A_{z}$, was calculated for each charge state, $z$, using ProTS Data software (Efeckta Technologies, Steamboat Springs, CO). The total ion count for a certain protein in a spectrum is given by:

$$
A_{\text {tot }}=\sum_{z=1}^{n} A_{z}
$$

where $n$ is the highest charge state observed. The average charge state $(\bar{Z})$ was calculated as a weighted average:

$$
\bar{Z}=\sum_{z=1}^{n} z \cdot A_{z} / A_{t o t}
$$

The ion counts and average charge state values were corrected for the following known instrumental biases. (Note: the spectra shown in the figures are raw data, but the calculated results in data plots employed the corrections.) The first correction relates to a decrease in signal when the gas flow is increased through the corona discharge part of the nozzle extension. Forcing gas through this corona discharge side-port necessarily reduces the vacuum-induced flow coming from the electrospray region, which leads to somewhat fewer electrospray ions entering the mass spectrometer (refer to Figure 1a). Control spectra were taken at each gas flow rate but without a corona discharge present. The $A_{\text {tot }}$ value for each of these control spectra was ratioed to $A_{\text {tot }}$ for the zero-flow spectrum to determine the fraction of ions lost for a given flow rate. The second type of correction corresponds to the preferential loss of faster-moving small ions in the orthogonal acceleration region of the spectrometer. The correction factor simply depends upon $(\mathrm{m} / \mathrm{z})^{1 / 2}$ [31]. The last type of correction involves the $\mathrm{m} / \mathrm{z}$ bias of the microchannel plate (MCP) detector. The efficiency with which an ion generates a secondary electron in the MCP has been shown to vary as follows [32-34]:

$$
\gamma_{\mathrm{e}}=\mathrm{C} \cdot m \cdot v^{4.4}
$$

where $m$ is mass, $v$ is velocity (calculated from the 4000 $\mathrm{V}$ acceleration potential), and $\mathrm{C}$ is a proportionality constant with a value of $10^{-24}$ and units that cancel the SI units of $m$ and $v$ to leave the unitless efficiency, $\gamma_{\mathrm{e}}$. The strong velocity dependence ( $>$ fourth power) means that slow-moving large $\mathrm{m} / \mathrm{z}$ ions produce significantly fewer ion counts than smaller $\mathrm{m} / \mathrm{z}$ ions.

\section{Results and Discussion}

\section{Corona Discharge CREMS}

Application of the charge reduction technique to electrospray ions at atmospheric pressure allows a control-

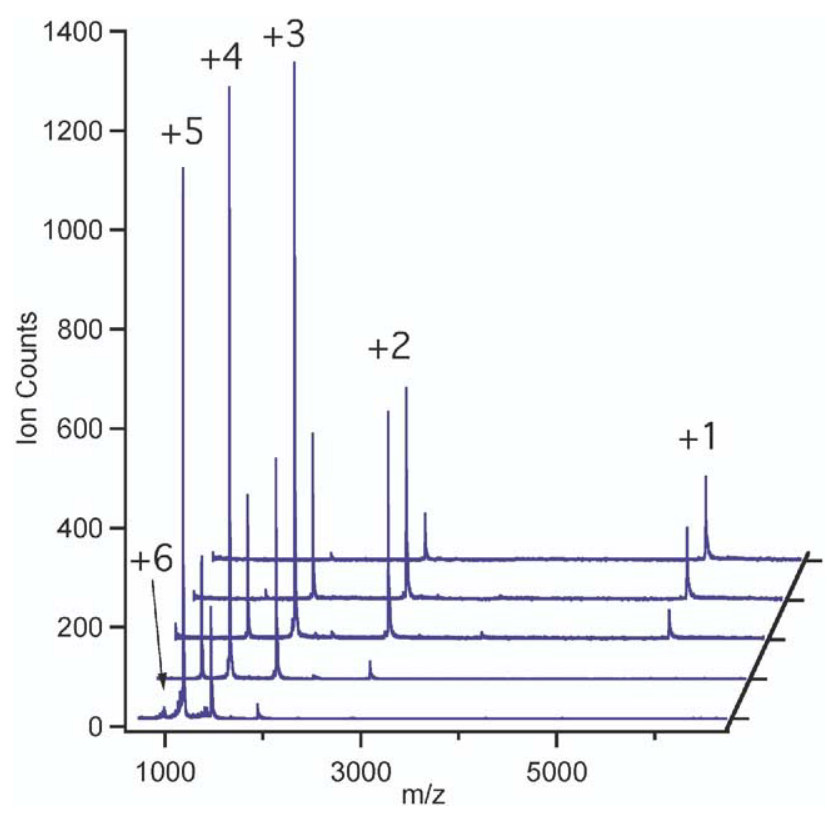

Figure 2. These mass spectra of insulin show increasing amounts of charge reduction from front to rear. The front trace is a normal electrospray spectrum (10 s acquisition). The next trace is a CREMS spectrum with minimal charge reduction that was acquired in $20 \mathrm{~s}$ with a corona discharge gas flow rate of $10 \mathrm{~mL} / \mathrm{min}$. The remaining CREMS spectra show further charge reduction and were obtained in $100 \mathrm{~s}$ with gas flow rates of 35, 60, and 200 $\mathrm{mL} / \mathrm{min}$, respectively.

lable shift to a lower charge state distribution. Figure 2 displays a normal electrospray mass spectrum of insulin as well as several CREMS spectra. Initially, charge states from +3 up to +6 are present, but charge reduction allows one to decrease these charges to the point where mostly +1 and +2 ions are observed. This change occurs because corona discharge generated anions abstract protons from the electrosprayed insulin ions, thereby reducing their charge [20, 21].

Changing the flow rate of gas through the corona discharge provides facile and reproducible control over the amount of charge reduction. As explained in the Experimental Section, anions such as $\mathrm{O}_{2}^{-}$are created by the corona discharge and then transported, via a gas stream through a small aperture, into the path of the electrosprayed ions (see Figure 1a). Figure 3 shows a plot of the average charge state $(\bar{Z})$ of insulin, a measure of the charge state distribution, as a function of this gas flow (circles, bottom axis). A gas flow of zero yields a value of $\bar{Z}=4.7$ (this data point corresponds to a normal ESI spectrum such as the front trace in Figure 2). Even though a corona discharge plasma is present, almost no charge reduction occurs without some gas flow through the aperture in the disk. As the flow rate increases, the average charge state decreases to where the +1 charge state predominates. For example, a gas flow rate of $200 \mathrm{~mL} / \mathrm{min}$ gives a value of $\bar{Z}=1.2$, which corresponds to the rear trace in Figure 2. It should be noted that completely charge neutralized ions $(z=0)$ are not detected in mass spectrometry and therefore are 


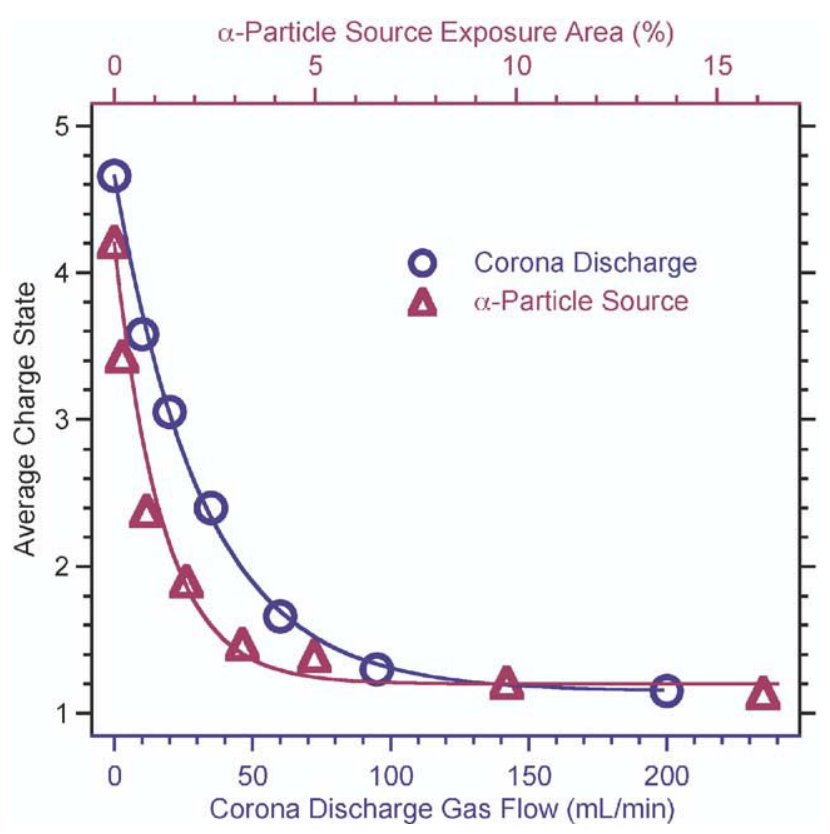

Figure 3. Two methods demonstrating control over the extent of charge reduction. In this plot, the amount of charge reduction is represented by the average charge state $(\bar{Z})$ of insulin. The circles correspond to gas flow rates through the corona discharge source. The triangles correspond to the percentage of the total area of the $\alpha$-particle source that was exposed to the electrosprayed ions. Error bars are smaller than the data points. The curves shown are exponential fits to the data.

not factored into the average charge state, which explains the asymptotic approach to $\bar{Z}=1$ instead of $\bar{Z}=$ 0 . Nonetheless, it is clear from the circle data points in Figure 3 that gas flow gives precise control over the amount of charge reduction. (Note: the $\alpha$-particle source data in Figure 3 is discussed later.)

Changing the gas flow through the corona discharge provides a convenient way of controlling the concentration of anions available to charge reduce electrosprayed ions via proton transfer reactions. McLuckey et al. have controlled charge reduction in their ion trap experiments with both the anion number density and by varying reaction time (usually tens to hundreds of milliseconds) [17, 18]. In our configuration, however, reaction time is constant. It is fixed by the travel time through the field-free region of the corona discharge nozzle extension and the nozzle itself, which was calculated in the Experimental section to be $\sim 10 \mathrm{~ms}$. Charge reduction reactions terminate after this time period because the electric field in the nozzle-skimmer region separates the positive and negative ions. Consequently, control of this atmospheric pressure CREMS technique relies upon controlling the gas-phase anion density rather than the reaction time.

Anion production by corona discharge can occur in either a current-limiting or a space-charge-limiting regime [35]. In previous work, a corona discharge shielded with wire mesh was located within the path of the electrosprayed ions, and charge reduction was controlled with an applied potential, which in turn varied the corona current (i.e., the current limiting regime) [21]. The alternate design presented here (Figure 1a) removes the intense electric fields of the corona from the path of the electrosprayed ions and thereby leads to higher signal intensities and more stable corona discharges. With this configuration, varying the applied potential (above the corona onset voltage) produces no change in the amount of charge reduction and very little change in corona current. This behavior is indicative of a plasma with higher ion density where space-charge effects limit the anion density and keep it constant over time [35].

Flowing gas through this space-charge limited plasma is an effective method of controlling the anion concentration available for reaction with the protein cations. As shown in Figure 3, the average charge state decays exponentially as a function of gas flow rate. This result is qualitatively consistent with pseudo-first-order kinetics:

$$
\left[(M+z H)^{z+}\right] \approx\left[(M+z H)^{z+}\right]_{0} \mathrm{e}^{-k t\left[A^{-}\right]}
$$

where $\left[(M+z H)^{z+}\right]$ is the protein cation concentration with the subscripted 0 indicating its initial concentration, $k$ is the reaction rate constant, $t$ is the time of reaction, and $\left[A^{-}\right]$is the anion concentration (present in large excess to apply the pseudo-first-order assumption). The classical situation for pseudo-first-order kinetics is an exponential decay curve when reactant concentration is plotted as a function of time [36]. In the present case, the results shown in Figure 3 differ from this classical plot in two respects. First, the average charge state plotted on the y-axis is a composite quantity that reflects the gas-phase concentration of several different charge states, not just a single reactant species. As shown elegantly by McLuckey, each of these charge states will exhibit differing reaction kinetics that depend upon the square of the charge state of the protein ion $[17,37,38]$. Second, the plot in Figure 3 shows the average charge state as a function of gas flow rate rather than as a function of reaction time. This difference is still consistent with eq 4, since a similar exponential relationship is obtained with either time or anion concentration as the independent variable (where the other one is held constant). The exponential behavior observed thus suggests that the anion concentration depends linearly upon the gas flow rate, which seems reasonable because the gas flow simply moves anions from the plasma region into the reaction chamber. A full kinetic analysis of this system is outside of the scope of the present paper and will be presented elsewhere. In the meantime, the results shown suggest strongly that pseudo-first-order kinetics give a good description of the behavior of this system, and that varying the gas flow through the corona discharge plasma does provide excellent control of the anion concentration and consequently the extent of charge reduction for electrosprayed protein ions. 


\section{Oxidation and Adduction Reactions}

The desired type of reaction during CREMS is proton transfer; other reactions will yield products with different masses and consequently decrease peak intensity. For instance, multiple peaks are observed in Figure 4a, which shows an expanded view of the +1 charge state of insulin after charge reduction. Along with the expected peak at 5734.6 $\mathrm{Da}$, additional peaks occur on the high mass side with an average spacing of $16 \mathrm{Da}$. The obvious culprit yielding this mass shift is an oxygen atom. Thus, it appears that some species generated in the corona discharge plasma lead to oxidation of the protein. Maleknia et al. have purposely caused protein oxidation with X-ray radiolysis [39] or a discharge from the electrospray tip $[40,41]$ to study protein structure with tandem mass spectrometry. For analysis of protein mixtures by charge reduction, however, oxidation is merely a deleterious side reaction that decreases the desired signal and increases spectral complexity.

These oxidation reactions can be prevented by addition of an organic vapor to the gas stream flowing through the corona discharge. A logical first attempt at preventing oxidation employed nitrogen gas, rather than air, as both the corona discharge gas and as the electrospray atmosphere. Unfortunately, oxidation was as bad or worse under these conditions (see Figure $4 b$ ), and similar results were observed for argon and carbon dioxide as well. Subsequently, various vapors were added to the gas stream flowing through the plasma in an attempt to produce species that are less reactive. As shown in Figure 4c and d, water vapor greatly increased the amount of oxidation, but methanol vapor eliminated it. Ethanol, acetonitrile, as well as a methanol/water vapor mixture all were as effective as methanol vapor at eliminating protein oxidation.

Despite solving the oxidation problem, methanol vapor in air still does not give the desired single peak for the charge reduced insulin spectrum due to adduction. The spectrum in Figure 4d shows adduct peaks at $M+63$ and $M+126$; note that they are also observed in the absence of methanol vapor (Figure 4a). The species forming these adducts is likely a $62 \mathrm{Da}$ anion. To
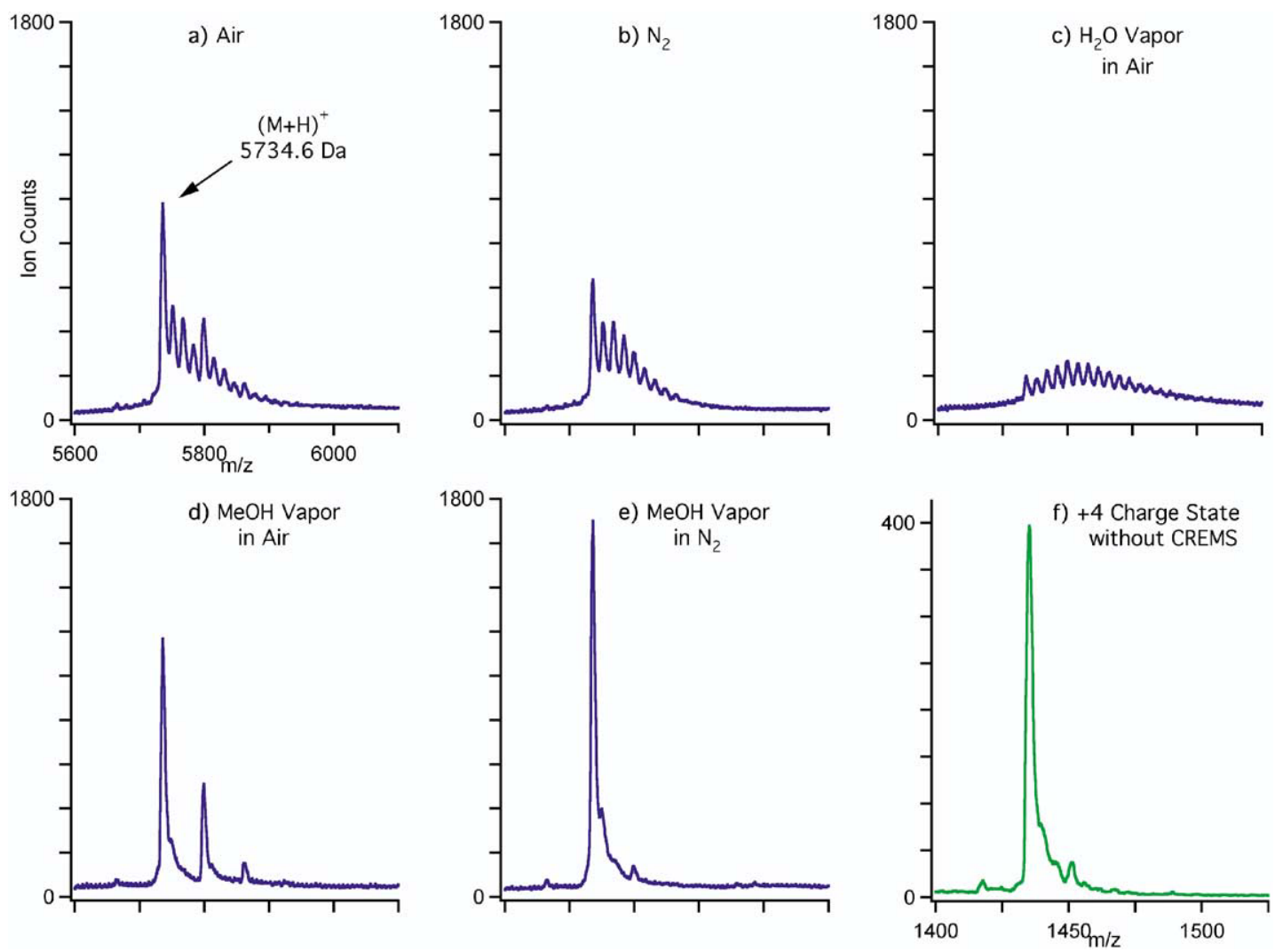

Figure 4. (a)-(e). These first five mass spectra have identical axes showing the +1 charge state of insulin obtained with CREMS. As noted in the figure, various gases and vapors were flowed through the corona discharge source. In addition to the expected 5734.6 Da peak, spectra (a), (b), and(c) contain multiple peaks with $\sim 16$ Da spacing attributable to oxidation of the protein. These oxidation peaks are not present in spectrum (d), but in that case nitrate adducts are observed with 63 Da spacing. Spectrum (e) shows that both oxidation and nitrate adduction are prevented by using methanol vapor in nitrogen gas. (f) An expanded view of the +4 charge state of insulin is shown for a spectrum obtained without charge reduction (5 s acquisition). Qualitatively, spectra (e) and (f) are nearly identical, which confirms that the spurious side reactions of CREMS have been eliminated. 
identify it, a negative ion mode spectrum was acquired when operating the corona discharge without performing electrospray. A strong peak at $61.983 \mathrm{Da}$ was observed and identified as the nitrate anion $\left(\mathrm{NO}_{3}^{-}\right)$, which has a theoretical mass of $61.988 \mathrm{Da}$. Nitrate is known to form in corona discharges in air because of the combination of nitrogen and oxygen forming $\mathrm{NO}$, which undergoes further oxidation to $\mathrm{NO}_{2}$ and eventually to $\mathrm{NO}_{3}^{-}$[28-30]. When this nitrate anion collides with an electrosprayed protein ion, it might abstract a proton and leave as $\mathrm{HNO}_{3}$ or it might form an adduct via ion attachment. Apparently, adduct formation is more energetically favorable in this case; even raising the nozzle-skimmer potential to $400 \mathrm{~V}$ did not disrupt this adduct. Others have observed attachment of certain anions to protein cations [42, 43]. A reasonable strategy for avoiding this particular adduct is to prevent nitrate from ever forming in the corona discharge by eliminating $\mathrm{O}_{2}$. Figure $4 \mathrm{e}$ shows the result from flowing methanol vapor in nitrogen gas through the corona discharge. This spectrum contains only very small peaks due to oxidation and nitrate adduction, and consequently the desired peak at $5734.6 \mathrm{Da}$ is more intense than in the previous spectra (Figure $4 a, b, c$, and d). In fact the spectrum is essentially identical to that of insulin without charge reduction (Figure 4f). Therefore, flowing an organic vapor in nitrogen gas through the corona discharge eliminates oxidation and adduction reactions in CREMS.

To gain further insight into the oxidation reactions, similar experiments were performed with a smaller peptide, which yielded resolved isotope peaks of the charge-reduced ions. Normal electrospray of the nonapeptide bradykinin gives predominantly doubly charged ions, which CREMS converts to singly charged ions. The rear trace in Figure 5 exhibits a CREMS spectrum of bradykinin where the base peak is the monoisotopic peak at 1060.6 Da and the isotope peaks are resolved. As evidenced by the peak clusters at multiples of $16 \mathrm{Da}$, oxidation reactions are occurring, which is expected since no organic vapor was added to the gas flowing through the corona discharge. The most intriguing aspect of this spectrum is the appearance of peaks at one and two Da lower than the monoisotopic peak. These noteworthy peaks are absent from spectra obtained (1) without CREMS (not shown) and (2) with CREMS employing methanol vapor to avoid oxidation (front trace in Figure 5). Furthermore, the intensity of these peaks roughly scales with the amount of oxidation observed. Therefore, the species reacting with bradykinin to produce these peaks is quite likely related to the oxidation chemistry occurring in the CREMS source.

Observation of these noteworthy peaks along with results from the vapor studies leads us to believe that hydroxyl radical $(\cdot \mathrm{OH})$ is the agent responsible for oxidation during CREMS. The hydroxyl radical is one of the most potent gas-phase oxidizing species known and accounts for much of the destruction of organic

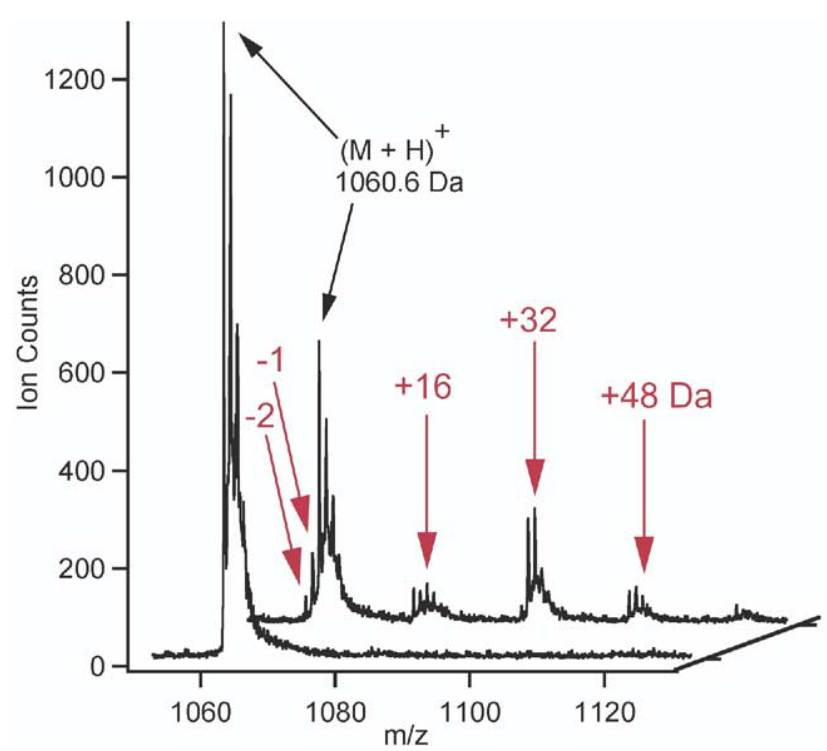

Figure 5. The front trace displays a CREMS spectrum of the +1 charge state of bradykinin. The spectrum reveals isotope peaks, but not oxidation or adduct peaks, because $\mathrm{MeOH}$ vapor in nitrogen gas was flowed through the corona discharge. The rear trace employed air instead, and consequently, it shows several peaks related to the reaction intermediates and products of oxidation (discussed in text).

compounds in the atmosphere [44]. Since it is not an ion, $\cdot \mathrm{OH}$ cannot be observed with mass spectrometry and so we will provide only indirect evidence of its existence. A common initial reaction between a hydroxyl radical and an organic compound involves abstraction of a hydrogen atom. A protein cation, for example, would undergo the following reaction:

$$
\mathrm{RH}^{z+}+\cdot \mathrm{OH} \rightarrow \mathrm{R}^{z+\cdot}+\mathrm{H}_{2} \mathrm{O}
$$

The resulting radical cation quite possibly can exist at atmospheric pressure for a few milliseconds before entering the vacuum system, thereby explaining the loss of one Dalton observed in Figure 5. In addition, the radical ion, $\mathrm{R}^{\mathrm{z+}}$, may lose another hydrogen due to weakened bonds next to the radical site, which would form an alkene and explain the peak at $-2 \mathrm{Da}$. Another intriguing aspect of the bradykinin spectrum is that the tallest oxidation related peaks occur at +31 and +32 , as opposed to +16 . This feature is also consistent with the known mechanism of hydroxyl radical induced oxidation in the atmosphere, namely attachment of $\mathrm{O}_{2}$ to the radical [44]:

$$
\mathrm{R}^{z+\cdot}+\mathrm{O}_{2} \rightarrow \mathrm{RO}_{2}^{z+}
$$

The $\mathrm{RO}_{2}^{\mathrm{z+}}$ ion explains the prominence of the +31 mass shift, and this ion may undergo further reactions to yield various products such as an aldehyde $(+14 \mathrm{Da})$, an alcohol (+16 Da), hydroperoxides (+32), and others. The mechanism proposed above pertains primarily to .OH attack in an alkyl region of the protein, but many 
other reactions are possible, notably reactions with aromatic, heterocyclic or sulfur-containing amino acid side-chains [39]. Consequently, a thorough analysis of reaction products is beyond the scope of this paper. Nonetheless, these hydroxyl radical reactions appear consistent with several aspects of the bradykinin spectrum. Other than reaction with $\cdot \mathrm{OH}$, it is difficult to find a plausible scenario for loss of $1 \mathrm{Da}$ from an electrosprayed peptide ion at atmospheric pressure, even though it is observed in vacuo with electron impact ionization.

Further indirect evidence for hydroxyl radical induced oxidation comes from the results of testing various vapors in the corona discharge. The likely source of $\cdot \mathrm{OH}$ is water vapor via a reaction with a high-energy species produced in the corona discharge, such as the singlet-D oxygen atom:

$$
\mathrm{O}\left({ }^{1} \mathrm{D}\right)+\mathrm{H}_{2} \mathrm{O} \rightarrow 2 \cdot \mathrm{OH}
$$

In a typical experiment, the electrospray is the source of water vapor. Adding water vapor directly to the gas flowing through the corona discharge greatly increases the amount of oxidation (Figure 4c), as expected from additional $\mathrm{OH}$ being produced by Rxn 7 . Conversely, organic vapors in the corona discharge, such as methanol, react with $\mathrm{O}\left({ }^{1} \mathrm{D}\right)$, or any other high-energy species, before it encounters the water vapor from the electrospray, thereby preventing hydroxyl radical formation and subsequent protein oxidation.

\section{CREMS Comparison: Corona Discharge versus $\alpha$-Particle Source}

Charge reduction of positive electrospray ions occurs easily with anions created by either a corona discharge or a radioactive $\alpha$-particle source. Figure 6 displays several CREMS spectra of insulin obtained with a ${ }^{210} \mathrm{Po}$ $\alpha$-particle source, and they are quite similar to the corona discharge ones in Figure 2. Interestingly, the $\alpha$-particle source operating in an atmosphere of air does not give rise to oxidation peaks, but the nitrate adducts are present. Evidently these $\alpha$-particles do not produce $\mathrm{O}\left({ }^{1} \mathrm{D}\right)$ in air and the resulting hydroxyl radical-induced oxidation. However, some oxidation is observed when employing the $\alpha$-particle source in a nitrogen atmosphere (data not shown). One possible explanation is that a high-energy nitrogen species is produced that usually reacts with $\mathrm{O}_{2}$, but in its absence produces $\cdot \mathrm{OH}$ from water vapor.

Control over the amount of charge reduction is accomplished by changing the area of the $\alpha$-particle source exposed to the electrospray ions in the nozzle extension (refer to Figure 1b). As shown in Figure 3 (triangles, top axis), the average charge state of insulin is decreased by $\alpha$-particle CREMS in a manner very similar to that by corona discharge (circles, bottom axis). It seems reasonable to assume that anion concentration in the chamber is directly proportional to the

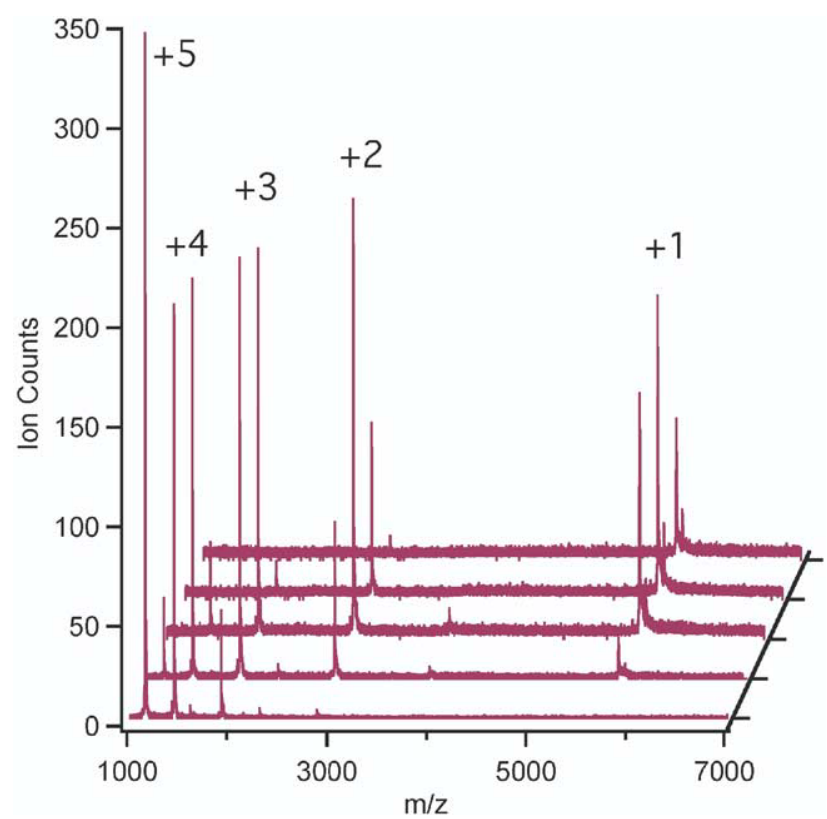

Figure 6. CREMS spectra of insulin utilizing an $\alpha$-particle source. As in Figure 2, the front trace was acquired in $10 \mathrm{~s}$ without charge reduction. The second trace is a CREMS spectrum obtained in $30 \mathrm{~s}$ using an $\alpha$-particle exposure area of $0.8 \%$. The remaining spectra employed exposure areas of $3.2,16$, and $65 \%$, all with $100 \mathrm{~s}$ acquisition times. As demonstrated by the rear trace, a high $\alpha$-particle exposure eliminates all but the +1 charge state.

$\alpha$-particle exposure area. Therefore, the exponential fit to the data again suggests pseudo-first-order kinetics of the ion/ion proton transfer reactions.

The $\alpha$-particle source with a large exposure area has the potential to generate higher anion densities than the corona discharge. By comparing the most chargereduced spectra between Figure 2 and Figure 6, one will notice that the $\alpha$-particle source can eliminate virtually all of the higher charge states leaving only +1 protein ions. The price for this result, however, is complete neutralization of many ions and consequently a smaller +1 peak than if some +2 ions were allowed to remain. In fact, using an $\alpha$-particle exposure area of $100 \%$ completely neutralized all ions, leaving a mass spectrum devoid of peaks. Although corona discharge CREMS could employ an increased flow rate to achieve further charge reduction, the electrospray signal does decrease significantly at high corona gas flow rates. Therefore, $200 \mathrm{~mL} / \mathrm{min}$ is a reasonable upper limit that produces as many +1 ions as higher flow rates, but it does leave some +2 ions as well.

One explanation for why the $\alpha$-particle source can produce a higher anion density is that it produces a bipolar neutralizing gas containing both positive and negative ions [45]. On the other hand, the corona discharge is a unipolar source of ions, and so anion density is limited by the space-charge effect mentioned earlier. Thus, the $\alpha$-particle source has the advantage for very extensive charge reduction, but the corona discharge source is usually adequate and has some 
other advantages. For instance, it avoids the licensing and handling of radioactive materials as well as the gradual decrease in $\alpha$-particle intensity over time (halflife of ${ }^{210} \mathrm{Po}=138$ days). Also, with the current designs the corona discharge gas flow provides more facile control over charge state distribution than the $\alpha$-particle exposure area, especially when minimal charge reduction is desired.

\section{Mass Discrimination}

Despite the advantages of charge reduction of proteins, a possible problem involves the decreased signal intensity (refer to Figure 2 and Figure 6). We have sought to determine the source of this signal fall-off that occurs with increasing $\mathrm{m} / \mathrm{z}$ for CREMS. The two most likely possibilities are ion losses during the charge reduction process and instrumental biases affecting the transmission and detection efficiencies of different $m / z$ ions (i.e., mass discrimination). Recently, our group ascertained that instrumental factors account completely for the mass fall-off observed in MALDI-TOF analysis of DNA oligomers [32]. Unfortunately, it is more difficult to measure or model ion transmission through each component of an ESI-oaTOF instrument because of the transport of ions from atmospheric pressure to the vacuum system, and also the additional ion optics such as an RF-only quadrupole for collisional cooling. Nonetheless, the data were corrected using measurements and models that were either straightforward or deemed most significant from prior work.

Three types of signal intensity corrections were employed: gas flow rate, $m / z$ bias in the orthogonal acceleration region of the TOF analyzer, and $m / z$ bias of the microchannel plate (MCP) detector. The uncorrected data in Figure 7 (squares) show that the total ion count does decrease dramatically as the average charge state is reduced by CREMS. Each of the three individual corrections is shown in the figure (see the Experimental section for quantitative details). Gas flow through the corona discharge nozzle extension tends to cause some ion losses except at very low flow rates, and so accounting for this effect generally increases the corrected ion count. The orthogonal acceleration into the time-offlight drift tube always favors higher $\mathrm{m} / \mathrm{z}$ ions, which means this oa-TOF correction decreases the corrected ion count of charge-reduced spectra. The MCP detector is much less efficient for the high $\mathrm{m} / \mathrm{z}$ ions in the CREMS spectra, as indicated by the large change in ion count when applying this detector correction.

Applying all three of the corrections to the data results in a much higher normalized total ion count (circles in Figure 7). The dashed line gives a visual representation of the expected signal if no ion losses or instrumental biases occurred. The corrected data follow the dashed line initially but then fall away from the line with more extensive charge reduction. This trend is expected since an increasing number of ions are completely neutralized and thereby avoid detection. The

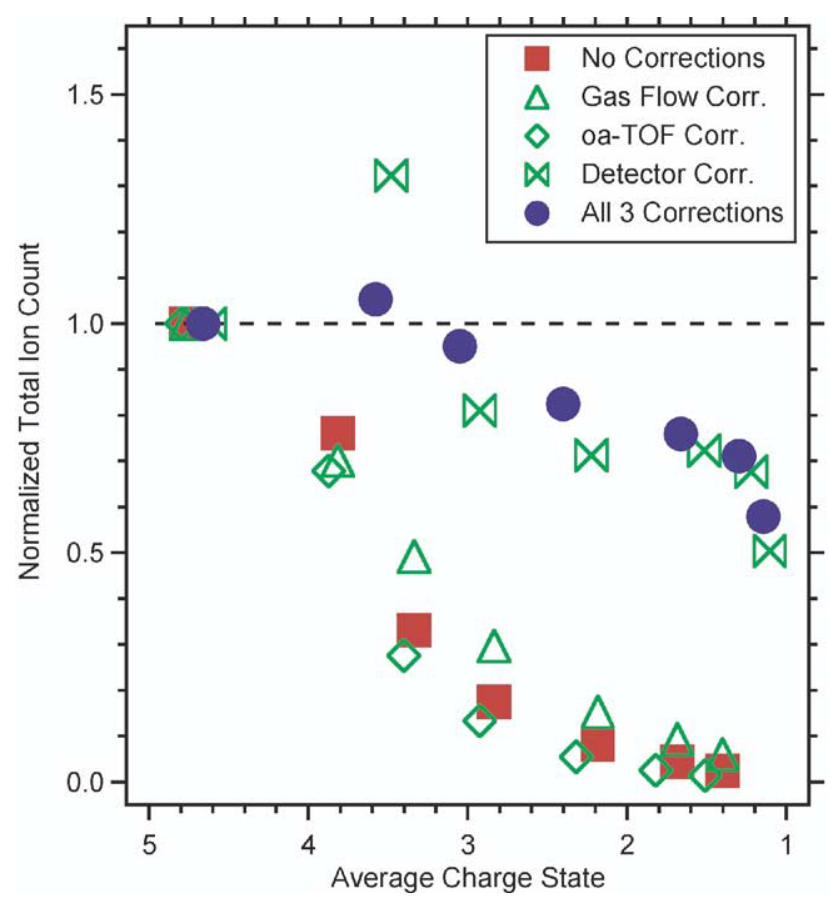

Figure 7. The signal intensity is plotted versus the average charge state $(\bar{Z})$. The results of three individual corrections are shown, as well as the combination of all three corrections. Each data point represents the sum of the ion counts from all of the charge states in a corona discharge CREMS spectrum of insulin. The data were normalized to the total ion count obtained without charge reduction (the leftmost data point).

approximate agreement between the corrected data and the expected signal suggests that we have accounted for the largest factors influencing the ion count from CREMS. However, changing the quadrupole RF amplitude or nozzle potential also impacts the transmission efficiency of various $\mathrm{m} / \mathrm{z}$ ions, and so the above results are not complete quantitative corrections for mass discrimination. Nonetheless, it is quite apparent that the inefficiency of the MCP detector at higher $\mathrm{m} / \mathrm{z}$ values leads to the majority of the signal decrease occurring with charge reduction. The relatively low acceleration potential of $4000 \mathrm{~V}$ for the Mariner spectrometer used in this work exacerbates this detector inefficiency issue. Increased acceleration would provide significant signal enhancement for the charge-reduced ions because the detection efficiency of the MCP depends upon the ion velocity to about the fourth power (refer to eq 3). Most other TOF instruments employ a higher acceleration potential than the Mariner or, alternatively, one could institute "post acceleration" after the mass analyzer. The data in Figure 7 demonstrate that most of the observed CREMS signal decrease is due to mass discrimination of the detector rather than ion losses caused by the CREMS source or by complete neutralization.

\section{CREMS of a Protein Sample Isolated from E. coli}

The insights and improvements to CREMS gained from the above studies have been applied to a real biological 
sample. A fraction that resulted from first ion-exchange and then reverse-phase separation of intact proteins from a whole cell lysate of E. coli was obtained from Dave Robinette and Morgan Giddings. A normal ESI spectrum of this sample is shown in Figure 8a. A poor spectrum such as this one is typical for an inhomogeneous protein sample obtained using electrospray with a bench top mass spectrometer. One does observe in Figure 8a some resolved charge state peaks that produce a small peak in the deconvolution spectrum (see inset). In contrast, charge reduction yields completely resolved charge state peaks and more pronounced peaks in the deconvolution spectra (Figure $8 b$ and c).

As discussed above, employing methanol vapor in nitrogen gas for the corona discharge CREMS improves the resulting spectra. First, a better signal-to-noise ratio is observed in Figure $8 \mathrm{c}\left(\mathrm{MeOH}\right.$ vapor in $\mathrm{N}_{2}$ ) compared to Figure $8 \mathrm{~b}$ where the corona discharge occurred in air. Second, a more accurate mass is obtained; the corona discharge in air leads to oxidation and adduction reactions that shift the main peak in the deconvoluted spectrum by more than one kilodalton (49.2 versus 48.0 $\mathrm{kDa})$.

This E. coli fraction appears to contain predominantly one protein, but actually a few other proteins are present. The normal ESI spectrum reveals only the 48 $\mathrm{kDa}$ protein. The CREMS spectrum, however, shows a few additional peaks. The peak at $3710 \mathrm{~m} / \mathrm{z}$ is likely due to a small protein that has been charge-reduced to +1 . Other small peaks in Figure 8c yield the small features at 41.2 and $43.6 \mathrm{kDa}$ in the inset. In an effort to learn more about this sample, a MALDI-TOF spectrum was acquired (not shown). By far the most prominent peaks were attributable to the +1 and +2 charge states of a $48.1 \mathrm{kDa}$ protein, which matches the CREMS results quite well. In addition, small peaks were observed at 43.6 and $53.0 \mathrm{kDa}$. Thus, the MALDI results confirm the presence and measured mass of two proteins observed by CREMS. But also, each technique found a protein or two absent in the results from the other, which is not uncommon with the somewhat complementary ionization mechanisms of MALDI and electrospray.

\section{Conclusions}

Charge reduction has been performed on electrosprayed protein mixtures to simplify the mass spectra. This technique revealed additional proteins in a sample derived from $E$. coli by both decreasing the number of charge state peaks for each protein as well as spreading them across a wider $\mathrm{m} / \mathrm{z}$ range. The extent of the charge reduction reactions was easily controlled by varying the anion concentration supplied to the electrosprayed protein cations. This anion density was determined either by the flow rate of gas through the corona discharge or by the exposed area of the $\alpha$-particle source. Flowing nitrogen gas containing an organic vapor through the corona discharge source prevented undesired oxidation and adduction reactions by avoiding the formation of

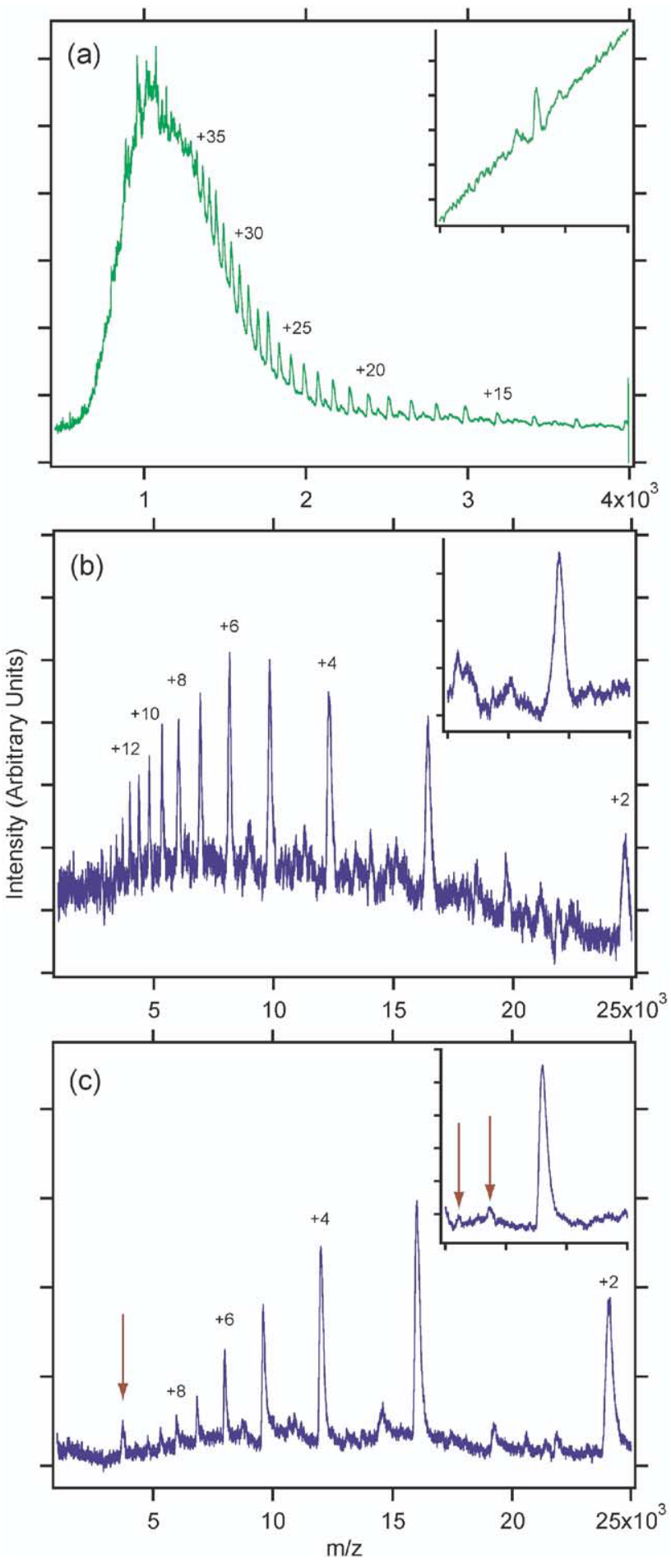

Figure 8. CREMS applied to a protein sample isolated from $E$. coli. (a) Mass spectrum obtained without charge reduction. (The inset shows the deconvolution spectrum with a small peak at 47.8 $\mathrm{kDa}$-note that all three insets are plotted from 40 to $55 \mathrm{kDa}$ ). (b) Corona discharge CREMS in air (deconvolution peak at $49.2 \mathrm{kDa}$ ). (c) Corona discharge CREMS with $\mathrm{MeOH}$ vapor in $\mathrm{N}_{2}$ (deconvolution peak at $48.0 \mathrm{kDa}$ ). The arrows indicate proteins not observed without CREMS.

hydroxyl radicals and nitrate ions. The inefficiency of the MCP detector for higher $-\mathrm{m} / \mathrm{z}$ ions was the major cause of the decreased signal intensity for charge- 
reduced spectra, whereas ion losses within the CREMS source or from neutralization appeared to be minimal. Charge reduction electrospray mass spectrometry at atmospheric pressure with the current nozzle extension design reduces spectral complexity for mixtures of intact proteins.

\section{Acknowledgments}

The authors are very grateful to Dave Robinette and Morgan Giddings for providing the E. coli protein sample. This work was supported by NIH grant R01HG001808 and NHLBI contract no. N01-HV-28,182.

\section{References}

1. Karas, M.; Bachmann, D.; Bahr, U.; Hillenkamp, F. MatrixAssisted Ultraviolet-Laser Desorption of Nonvolatile Compounds. Int. J. Mass Spectrom. Ion Processes 1987, 78, 53-68.

2. Hillenkamp, F.; Karas, M.; Beavis, R. C.; Chait, B. T. MatrixAssisted Laser Desorption Ionization Mass-Spectrometry of Biopolymers. Anal. Chem. 1991, 63, A1193-A1202.

3. Fenn, J. B.; Mann, M.; Meng, C. K.; Wong, S. F.; Whitehouse, C. M. Electrospray Ionization for Mass-Spectrometry of Large Biomolecules. Science 1989, 246, 64-71.

4. Smith, R. D.; Loo, J. A.; Loo, R. R. O.; Busman, M.; Udseth, H. R. Principles and Practice of Electrospray Ionization-MassSpectrometry for Large Polypeptides and Proteins. Mass Spectrom. Rev. 1991, 10, 359-451.

5. Smith, R. D.; Cheng, X.; Bruce, J. E.; Hofstadler, S. A.; Anderson, G. A. Trapping, Detection, and Reaction of Very Large Single Molecular-Ions by Mass-Spectrometry. Nature 1994, 369, 137-139.

6. Stephenson, J. L., Jr.; McLuckey, S. A. Ion/Ion Reactions for Oligopeptide Mixture Analysis: Application to Mixtures Comprised of 0.5-100 kDa Components. J. Am. Soc. Mass Spectrom. 1998, 9, 585-596.

7. Hayter, J. R.; Robertson, D. H. L.; Gaskell, S. J.; Beynon, R. J. Proteome Analysis of Intact Proteins in Complex Mixtures. Mol. Cell. Proteomics 2003, 2, 85-95.

8. Li, W. Q.; Hendrickson, C. L.; Emmett, M. R.; Marshall, A. G. Identification of Intact Proteins in Mixtures by Alternated Capillary Liquid Chromatography Electrospray Ionization and LC ESI Infrared Multiphoton Dissociation Fourier Transform Ion Cyclotron Resonance Mass Spectrometry. Anal. Chem. 1999, 71, 4397-4402.

9. Lee, S. W.; Berger, S. J.; Martinovic, S.; Pasa-Tolic, L.; Anderson, G. A.; Shen, Y. F.; Zhao, R.; Smith, R. D. Direct Mass Spectrometric Analysis of Intact Proteins of the Yeast Large Ribosomal Subunit Using Capillary LC/FTICR. Proc. Natl. Acad. Sci. U.S.A. 2002, 99, 5942-5947.

10. Meng, F. Y.; Cargile, B. J.; Patrie, S. M.; Johnson, J. R.; McLoughlin, S. M.; Kelleher, N. L. Processing Complex Mixtures of Intact Proteins for Direct Analysis by Mass Spectrometry. Anal. Chem. 2002, 74, 2923-2929.

11. McLuckey, S. A.; Stephenson, J. L., Jr. Ion/Ion Chemistry of High-Mass Multiply Charged Ions. Mass Spectrom. Rev. 1998, 17, 369-407.

12. He, M.; McLuckey, S. A. Charge Permutation Reactions in Tandem Mass Spectrometry. J. Mass Spectrom. 2004, 39, 12311259.

13. Pitteri, S. J.; McLuckey, S. A. Recent Developments in the Ion/Ion Chemistry of High-Mass Multiply Charged ions. Mass Spectrom. Rev. 2005, Published online,

14. Ikonomou, M. G.; Kebarle, P. An Ion-Source with which Ions Produced by Electrospray can be Subjected to Ion
Molecule Reactions at Intermediate Pressures (10-100 Torr)-Deprotonation of Polyprotonated Peptides. Int. J. Mass Spectrom. Ion Processes 1992, 117, 283-298.

15. McLuckey, S. A.; Vanberkel, G. J.; Glish, G. L. Reactions of Dimethylamine with Multiply Charged Ions of Cytochrome-c. J. Am. Chem. Soc. 1990, 112, 5668-5670.

16. Loo, R. R. O.; Smith, R. D. Investigation of the Gas-Phase Structure of Electrosprayed Proteins Using Ion-Molecule Reactions. J. Am. Soc. Mass Spectrom. 1994, 5, 207-220.

17. Stephenson, J. L., Jr.; McLuckey, S. A. Ion/Ion Reactions in the Gas Phase: Proton Transfer Reactions Involving MultiplyCharged Proteins. J. Am. Chem. Soc. 1996, 118, 7390-7397.

18. Reid, G. E.; Shang, H.; Hogan, J. M.; Lee, G. U.; McLuckey, S. A. Gas-Phase Concentration, Purification, and Identification of Whole Proteins from Complex Mixtures. J. Am. Chem. Soc. 2002, 124, 7353-7362.

19. Scalf, M.; Westphall, M. S.; Krause, J.; Kaufman, S. L.; Smith, L. M. Controlling Charge States of Large Ions. Science 1999, 283, 194-197.

20. Scalf, M.; Westphall, M. S.; Smith, L. M. Charge Reduction Electrospray Mass Spectrometry. Anal. Chem. 2000, 72, 52-60.

21. Ebeling, D. D.; Westphall, M. S.; Scalf, M.; Smith, L. M. Corona Discharge in Charge Reduction Electrospray Mass Spectrometry. Anal. Chem. 2000, 72, 5158-5161.

22. Ebeling, D. D.; Westphall, M. S.; Scalf, M.; Smith, L. M. A Cylindrical Capacitor Ionization Source: Droplet Generation and Controlled Charge Reduction for Mass Spectrometry. Rapid Commun. Mass Spectrom. 2001, 15, 401-405.

23. Schneider, B. B.; Baranov, V. I.; Javaheri, H.; Covey, T. R. Particle Discriminator Interface for Nanoflow ESI-MS. J. Am. Soc. Mass Spectrom. 2003, 14, 1236-1246.

24. Tanner, S. D.; Douglas, D. J.; French, J. B. Gas and Ion Dynamics of a 3-Aperture Vacuum Interface for InductivelyCoupled Plasma-Mass Spectrometry. Appl. Spectrosc. 1994, 48, 1373-1378.

25. Lu, Q.; Koropchak, J. A. Corona Discharge Neutralizer for Electrospray Aerosols Used with Condensation Nucleation Light-Scattering Detection. Anal. Chem. 2004, 76, 5539-5546.

26. Loeb, L. B. Recent Developments in Analysis of the Mechanisms of Positive Coronas in Air. J. Appl. Phys. 1948, 19, 882-897.

27. Carroll, D. I.; Dzidic, I.; Horning, E. C.; Stillwell, R. N. Atmospheric-Pressure Ionization Mass-Spectrometry. Appl. Spectrosc. Rev. 1981, 17, 337-406.

28. Yamashita, M.; Fenn, J. B. Negative-Ion Production with the Electrospray Ion-Source. J. Phys. Chem. 1984, 88, 4671-4675.

29. Nikolaev, E.; Riter, L. S.; Laughlin, B. C.; Handberg, E.; Cooks, R. G.Trace Analysis of Organics in Air by Corona Discharge Atmospheric Pressure Ionization Using an Electrospray Ionization Interface. Eur. J. Mass Spectrom. 10, 2004, 197-204.

30. Skalny, J. D.; Mikoviny, T.; Matejcik, S.; Mason, N. J. An Analysis of Mass Spectrometric Study of Negative Ions Extracted from Negative Corona Discharge in air. Int. J. Mass Spectrom. 2004, 233, 317-324.

31. Guilhaus, M.; Selby, D.; Mlynski, V. Orthogonal Acceleration Time-of-Flight Mass Spectrometry. Mass Spectrom. Rev. 2000, 19, 65-107.

32. Chen, X.; Westphall, M. S.; Smith, L. M. Mass Spectrometric Analysis of DNA Mixtures: Instrumental Effects Responsible for Decreased Sensitivity with Increasing Mass. Anal. Chem. 2003, 75, 5944-5952.

33. Geno, P. W.; Macfarlane, R. D. Secondary-Electron Emission Induced by Impact of Low-Velocity Molecular-Ions on a Microchannel Plate. Int. J. Mass Spectrom. Ion Processes 1989, 92, 195-210.

34. Westmacott, G.; Frank, M.; Labov, S. E.; Benner, W. H. Using a Superconducting Tunnel Junction Detector to Measure the 
Secondary Electron Emission Efficiency for a Microchannel Plate Detector Bombarded by Large Molecular Ions. Rapid Commun. Mass Spectrom. 2000, 14, 1854-1861.

35. Busman, M.; Sunner, J.; Vogel, C. R. Space-Charge-Dominated Mass-Spectrometry Ion Sources-Modeling and Sensitivity. J. Am. Soc. Mass Spectrom. 1991, 2, 1-10.

36. Houston, P. L. Chemical Kinetics and Reaction Dynamics; McGraw-Hill: Boston, 2001; pp 34-47.

37. Stephenson, J. L., Jr.; Van Berkel, G. J.; McLuckey, S. A. Ion-Ion Proton Transfer Reactions of Bio-Ions Involving Noncovalent Interactions: Holomyoglobin. J. Am. Soc. Mass Spectrom. 1997, 8, 637-644.

38. McLuckey, S. A.; Stephenson, J. L., Jr.; Asano, K. G. Ion/Ion Proton-Transfer Kinetics: Implications for Analysis of Ions Derived from Electrospray of Protein Mixtures. Anal. Chem. 1998, 70, 1198-1202.

39. Maleknia, S. D.; Brenowitz, M.; Chance, M. R. Millisecond Radiolytic Modification of Peptides by Synchrotron X-rays Identified by Mass Spectrometry. Anal. Chem. 1999, 71, 3965-3973.
40. Wong, J. W. H.; Maleknia, S. D.; Downard, K. M. Study of the Ribonuclease-S-Protein-Peptide Complex Using a Radical Probe and Electrospray Ionization Mass Spectrometry. Anal. Chem. 2003, 75, 1557-1563.

41. Maleknia, S. D.; Chance, M. R.; Downard, K. M. ElectrosprayAssisted Modification of Proteins: A Radical Probe of Protein Structure. Rapid Commun. Mass Spectrom. 1999, 13, 2352-2358.

42. Mirza, U. A.; Chait, B. T. Effects of Anions on the Positive Ion Electrospray Ionization Mass Spectra of Peptides and Proteins. Anal. Chem. 1994, 66, 2898-2904.

43. Stephenson, J. L., Jr.; McLuckey, S. A. Gaseous Protein Cations are Amphoteric. J. Am. Chem. Soc. 1997, 119, 1688-1696.

44. Seinfeld, J. H.; Pandis, S. N. Atmospheric Chemistry and Physics: From Air Pollution to Climate Change; Wiley: New York, 1998; pp 234-336.

45. Cooper, D. W.; Reist, P. C. Neutralizing Charged Aerosols with Radioactive Sources. J. Colloid Interface Sci. 1973, 45, $17-26$. 